\title{
Feeding practice and associated factors among infants and children with common childhood illness at Dabat Health and Demographic surveillance System site: A community based cross-sectional study
}

Gashaw Andargie Bikes

University of Gondar

Amare Tariku

University of Gondar

Molla Mesele Wassie

University of Gondar

Solomon Mekonnen

University of Gondar

Esmeal Ali Muhammad

University of Gondar

Aysheshim Kassahun Belew:

University of Gondar

Zegeye Abebe

University of Gondar

Azeb Atnafu Gete:

University of Gondar

Melkie Edris Yesuf

University of Gondar

Tadesse Awoke

University of Gondar

Abel Fekadu

University of Gondar

Ejigu Gebeye

University of Gondar

Yigzaw Kebede

University of Gondar

Kassahun Alemu

University of Gondar 


\section{Abebaw Addis}

University of Gondar

Kindie Fentahun

University of Gondar

Mekonnen Sisay

University of Gondar

Kedir Abdella

University of Gondar

Melkamu Tamir Hunegnaw ( $\square$ melkamutamir@gmail.com )

University of Gondar,Ethiopia

Research article

Keywords: Meal frequency, dietary diversity, Dabat HDSS, Ethiopia

Posted Date: March 2nd, 2020

DOI: https://doi.org/10.21203/rs.3.rs-15653/v1

License: (c) (1) This work is licensed under a Creative Commons Attribution 4.0 International License.

Read Full License 


\section{Abstract}

Background: Optimal feeding practices are recommended to improve nutritional status and prevent the common childhood illnesses. Studies on the feeding practice of children including meal frequency and dietary diversity are scarce in children with illness. Therefore, we aimed to assess feeding practice and its associated factors among children aged 6-59 months in Dabat Health and Demographic Surveillance System (HDSS) site, northwest Ethiopia.

Methods : A community based cross-sectional study was conducted in Dabat HDSS site from February to June 2016 on 1,174 mother-child pairs. A multistage stratified sampling followed by a systematic random sampling technique was employed to select the study participants. A binary logistic regression model was fitted to identify factors associated with children's feeding practice. A crude odds ratio and adjusted odds ratio with its $95 \%$ confidence intervals $(\mathrm{Cl})$ were calculated to see the strength of association and significance of the identified factors with feeding practices.

Result: The overall prevalence of a minimum dietary diversity practice and a minimum meal frequency was $27 \%(95 \% \mathrm{Cl}: 24.4,29.7)$ and $83.7 \%(95 \% \mathrm{Cl}: 91.7,86.0)$, respectively. Having antenatal care (ANC) service $(A O R=1.6 ; 95 \% \mathrm{Cl}: 1.18,2.27)$ and institutional delivery $(\mathrm{AOR}=2.6 ; 95 \% \mathrm{Cl}: 1.85,3.55)$ increased odds of an adequate dietary diversity in children. On the contrary, the odds of practicing an adequate dietary diversity was lower among a household obtained food from home gardens ( $A O R=0.4 ; 95 \% \mathrm{Cl}$ : $0.29,0.52)$ and currently breast fed children (AOR $=0.6 ; 95 \% \mathrm{Cl}: 0.42,0.78)$. The probability of having a minimum meal frequency was decreased among children in the first two years of age (AOR $=0.1 ; 95 \% \mathrm{Cl}$ : $0.06,0.15)$, however increased in children who fed alone (AOR $=1.9 ; 95 \% \mathrm{Cl}: 1.29,2.69)$.

Conclusion: The proportion of children who received the minimum dietary diversity was low while the proportion of children who obtained minimum meal frequency was good. Providing a health and nutrition counseling on Infant and Young Child Feeding during maternal ANC services and delivery period are recommended for achieving the recommended dietary practices.

\section{Background}

Appropriate feeding practices during and after illness are important measures to meet nutritional requirements of infants and children[1]. These children, requires a variety of foods and energy-rich food groups in the diet to ensures an adequate intake of essential nutrients and for growth and development[2]. Achieving the minimum dietary diversity for children aged 6-59 months is critical as they require energy and nutrient-dense foods for their physical, mental growth and development[3]. The World Health Organization (WHO) develops a Guiding Principles for Complementary feeding of breastfed and non breastfed children of an optimal feeding practices during and after common childhood illnesses to prevent a rapid deterioration of nutritional status associated with illness/convalescence as part of the WHO-led Global Strategy for the Integrated Management of Childhood IIInesses [4, 5]. 
Evidences indicate that, most under-nutrition in low and middle income countries occurs during young children as a result of suboptimal breastfeeding and complementary feeding practices[6, 7]. More importantly, children's nutritional status can deteriorate rapidly following illness, if the additional diversified nutrient requirements associated with illness are not met and nutrients are diverted to immune response against infection[8,9]. Despite the global progress in reducing child mortality over the past few decades, an estimated 5.4 million under 5 children died in 2017 and roughly half of these deaths occurred in sub-Saharan Africa [10]. More than $50 \%$ of child deaths are attributed to common childhood illness, of these $60 \%$ were attributed to malnutrition that associated with an inappropriate child feeding practice [11-13]. In Ethiopia, $38 \%$ of children are stunted, $10 \%$ are wasted, and $24 \%$ are underweight which informed the occurrence of both acute and chronic under nutrition. Only $7 \%$ and $47.7 \%$ of children received the minimum dietary diversity and meal frequency, respectively[14].

The cause of inappropriate feeding practices is multi-factorial and has diverse contributing factors[15]. Socio-economic and demographic characteristics, household family size, cultural and traditional beliefs were identified in previous studies $[16,17]$. However, those studies were not specifically focused on meal frequency and dietary diversity practices among children who had common childhood illnesses.

Even if the United Nations designs Sustainable Development Goals (SDGs) by the 2015 to decrease the deaths of under-5 children, as low as 25 per 1000 live births in every country by 2030 [18], and Ethiopia has been implementing strategies like the 2004 National Strategy for Infant and Young Child Feeding (IYCF) and the 2008 National Nutrition Program to improve the levels of child feeding practices[19, 20], malnourished children, particularly those with severe acute malnutrition, have a higher risk of death from common childhood illness such as diarrhea, pneumonia, and malaria and Ethiopia still in the list of nations with low levels of meal frequency and poor dietary diversity practices. Therefore, we aimed to assess the magnitude and factors associated with minimum meal frequency and dietary diversity practices among children aged 6-59 months who had an illness.

\section{Methods}

Study setting and design

This study was based on the baseline survey of five-year project entitled 'Establishing Nutrition Surveillance system and piloting intervention' the baseline survey was conducted from February to June 2016 in Dabat HDSS site which located in Dabat district, northwest Ethiopia. The HDSS site covers a total of 13 kebeles (9 rural and 4 urban kebeles, smallest administration unit in Ethiopia) with a total of 17,000 households and 69,468 populations. The kebeles under the surveillance site were selected randomly, and by taking into account of all the ecological zones (high land, middle land, and low land). Dabat HDSS is a full member of the International Network of Demographic Evaluation of Populations and Their Health (INDEPTH), a network of 44 HDSSs from the Global South.

The baseline survey included all children under five years, adolescents, pregnant and lactating mothers living in the Dabat HDSS site. Particularly, data on nutrition, health status and service utilization of 
children were gathered. For this study, we considered all children aged 6-59 months with illness. Child illness was ascertained asking the mothers whether her child had any illness within two weeks prior to data collection.

Sample size and sampling procedure

A minimum sample size for the study was estimated using single population proportion formula using Epi-info version 7 by considering the following assumptions: $38 \%$ as the proportion of adequate dietary diversity among children aged 6-59 months[21], 95\% level of confidence, $4 \%$ margin of error and 10\% non-response rate. Finally, we got 617 . Nevertheless, to increase the power of the study we have included all children $(1,174)$ aged 6-59 months with reported illness in the previous two weeks.

Data collection tool and procedure

The baseline survey questionnaire was initially prepared in English and translated into Amharic and retranslated to English with language and public health experts to check the consistency. The questionnaire was pretested in one kebele out of the kebeles under the HDSS site. Experienced 38 data collectors and seven field supervisors who have been permanently working HDSS site were involved in the data collection process. Three days training on interviewing technique and data collection process was given to data collectors and supervisors. Supervisors checked the overall baseline survey on a daily basis.

Assessment of dietary diversity and meal frequency

Assessment of the dietary diversity score (DDS) of each child was started by asking the mother to list all food consumed by the child in the last 24 hours preceding the survey. Then, the reported food items were classified into seven food groups: grains, roots and tubers; legumes and nuts; dairy products (milk, yogurt, cheese); flesh foods (meat, fish, poultry and liver/organ meats); eggs; vitamin-A-rich fruits and vegetables; other fruits and vegetables. Children who got four or more food groups were classified as meeting the minimum dietary diversity; otherwise, they were considered as getting inadequate minimum dietary diversity [2]. A meal frequency of the child was assessed by asking the mother how many times the child took solid, semi-solid or soft foods in the last 24 hours preceding the survey. Accordingly, two or more times for breastfed infants 6 to 8.9 months of age, three or more times for breastfed children 9 to 23.9 months and four times for non-breastfed children 6 to 59 months were considered as children who received a minimum meal frequency [2].

Data processing and analysis

Data were entered into EPI INFO version 7 and analyzed using a Statistical Package for Social Sciences (SPSS) version 20. Descriptive statistics, including frequencies and proportions were used to summarize the study variables. A binary logistic regression model was fitted to identify factors associated with a minimum meal frequency and a minimum dietary diversity practices. Variables with $\mathrm{P}-\mathrm{Values}$ of $<0.2$ in the bi-variable analysis were entered in to the multivariable analysis to control for the possible effect of 
confounders. The Adjusted Odds Ratio (AOR) with a $95 \%$ of confidence intervals was used to examine the strength of association, and a $\mathrm{P}-$ Values $\leq 0.05$ was used to declare the statistical significance in the multivariable analysis.

\section{Results}

A total of 1,174 children aged 6-59 months who had at least the common childhood illness in the last two weeks were included for analysis. The mean age $( \pm S D)$ of the mothers were $29.06( \pm 6.58$ years) and 1,017(86.6\%) were married (Table 1).

Table 1

Parental level Socio-demographic characteristics among children aged 6-59 months at Dabat HDSS, site northwest

Ethiopia, 2016

\begin{tabular}{|lll|}
\hline Variables & Frequency & Percentage \\
\hline Maternal religion & & \\
\hline Orthodox Christian & 1150 & 98 \\
\hline Muslim & 24 & 2 \\
\hline Residence & & \\
\hline Urban & 133 & 11.3 \\
\hline Rural & 1041 & 88.7 \\
\hline Marital status & & \\
\hline Married & 1017 & 86.6 \\
\hline Single & 109 & 9.3 \\
\hline Divorced & 30 & 26 \\
\hline Windowed & 10 & 0.9 \\
\hline Separated & 8 & 0.7 \\
\hline Maternal educational status & & \\
\hline Unable to read and write & 899 & 76.6 \\
\hline Abel to read and write & 35 & 3.0 \\
\hline Primary education & 166 & 14.1 \\
\hline Secondary education & 61 & 5.2 \\
\hline Higher education & 13 & 1.1 \\
\hline
\end{tabular}


A quarter $(26.0 \%)$ of children were born in a health institution, and 696(59.3\%) were currently breastfed. Eight hundred forty (71.6\%) subjects initiated complementary feeding between 6 to 8 months. Two-thirds, $768(65.4 \%)$, of the mothers attended at least one ANC follow-up and 108 (9.6\%) of the mothers had PNC visits. One-fourth (28.8\%) of the children had watery diarrhea prior to 2 weeks before data collection (Table 2). 
Table 2

Socio demographic and health care level related characteristics among children aged 6-59 months at Dabat HDSS site, northwest Ethiopia, 2016

\begin{tabular}{|c|c|c|}
\hline Variables & Frequency & Percentage \\
\hline \multicolumn{3}{|l|}{ Age of the child } \\
\hline $6-23$ & 517 & 44.0 \\
\hline $24-59$ & 657 & 56.0 \\
\hline \multicolumn{3}{|l|}{ Sex of the child } \\
\hline Female & 595 & 50.7 \\
\hline Male & 579 & 49.3 \\
\hline \multicolumn{3}{|l|}{ ANC service } \\
\hline Yes & 777 & 66.2 \\
\hline No & 397 & 33.8 \\
\hline \multicolumn{3}{|l|}{ Birth place } \\
\hline Health institution & 305 & 26.0 \\
\hline Home & 869 & 74.0 \\
\hline \multicolumn{3}{|l|}{ Birth order } \\
\hline First & 160 & 13.6 \\
\hline Second to fifth & 698 & 59.5 \\
\hline Above fifth & 316 & 26.9 \\
\hline \multicolumn{3}{|c|}{ Currently breast feed } \\
\hline Yes & 696 & 59.3 \\
\hline No & 478 & 40.7 \\
\hline \multicolumn{3}{|c|}{ Postnatal care service } \\
\hline Yes & 108 & 9.6 \\
\hline No & 1066 & 90.8 \\
\hline \multicolumn{3}{|c|}{ Time of complementary feeding initiation } \\
\hline$<6$ months & 87 & 7.4 \\
\hline 6-8months & 840 & 71.6 \\
\hline
\end{tabular}




\begin{tabular}{|lll|}
\hline Variables & Frequency & Percentage \\
\hline 9months and above & 247 & 21.0 \\
\hline Source of complementary food & & \\
Home Garden & 838 & 71.4 \\
Market & 336 & 28.6 \\
Current illness & & \\
Watery diarrhea & 303 & 25.8 \\
Dysentery & 97 & 8.3 \\
RTI & 367 & 31.3 \\
AFI & 374 & 31.9 \\
Ear problem & 35 & 2.7 \\
\hline
\end{tabular}

Dietary diversity and meal frequency practices.

The proportion of children who received a minimum dietary diversity was $27 \%$ (95\% Cl: $24.4,29.7 \%)$. A meal frequency among breastfed and non-breastfeed children was 83.9\% (95\% Cl: 81.9, 85.9\%) and $57.8 \%$ (95\% Cl: $54.9,60.5 \%$ ), respectively. Cereals and roots were provided to the greatest portion of the children (95.7\%), and only 24 (2\%) of the children were given vitamin A source foods. (Fig. 1)

Factors associated with minimum dietary diversity and minimum meal frequency in children common childhood illness.

The result of multivariate analysis revealed that ANC visits, food source and place of birth were statistically and independently associated with dietary diversity practice in the study area. Similarly, child age and preparation of complementary foods were statistically associated with a minimum meal frequency.

Accordingly, children whose mothers had ANC visit had 1.6 times (AOR $=1.64 ; 95 \% \mathrm{Cl}: 1.18,2.27)$ more likely to have diversified diet compared to their counterparts. Similarly, higher odds of diversified diet were noted among sick children whose mothers gave birth at health facility ( $\mathrm{AOR}=2.6 ; 95 \% \mathrm{Cl}: 1.85,3.55)$, whereas and children from households using home gardening as prime food source had lower odds of adequate dietary diversity than children from households securing food mainly through purchasing from market (Table 3). 
Table 3

Bivariate and multivariate analysis of factors associated with minimal dietary diversity among children aged 6-59 months at Dabat HDSS, site northwest Ethiopia, 2016

\begin{tabular}{|c|c|c|c|c|}
\hline \multirow[t]{2}{*}{ Variables } & \multicolumn{2}{|c|}{ Minimum dietary diversity } & \multirow[t]{2}{*}{ Crude OR(95\%Cl) } & \multirow[t]{2}{*}{ Adjusted OR(95\% Cl) } \\
\hline & Adequate & Inadequate & & \\
\hline \multicolumn{5}{|l|}{ Food security } \\
\hline Secured & 234 & 677 & $0.75(0.99-1.80)$ & $0.80(0.57,1.08)$ \\
\hline In secured & 83 & 180 & 1 & \\
\hline \multicolumn{5}{|l|}{ Food source } \\
\hline Home garden & 169 & 669 & $0.32(0.24,0.42)$ & $0.40(0.29,0.52) \star \star$ \\
\hline Market & 148 & 188 & 1 & 1 \\
\hline \multicolumn{5}{|l|}{ ANC service } \\
\hline Yes & 245 & 532 & $2.20(1.61,2.91)$ & $1.60(1.18,2.27)^{\star \star}$ \\
\hline No & 72 & 333 & 1 & 1 \\
\hline \multicolumn{5}{|c|}{ Complementary food preparation } \\
\hline Prepared alone & 105 & 211 & $1.51(1.14,2.00)$ & $1.30(0.95,1.81)$ \\
\hline Prepared with others & 212 & 646 & 1 & \\
\hline \multicolumn{5}{|l|}{ Current breast feeding } \\
\hline Yes & 176 & 520 & $0.80(0.62,1.05)$ & $0.60(0.43,0.78)^{\star \star}$ \\
\hline No & 141 & 337 & 1 & \\
\hline \multicolumn{5}{|l|}{ Place of birth } \\
\hline Health institutions & 141 & 164 & $3.40(2.55,4.47)$ & $2.60(1.85,3.55)^{\star \star}$ \\
\hline Home & 176 & 693 & 1 & \\
\hline
\end{tabular}

On the other hand, increased odds of having adequate meal frequency was illustrated among ill children whose mothers prepared food for their child alone (AOR $=1.9 ; 95 \% \mathrm{Cl}: 1.28,2.69)$ compared to counterparts. However, the odds of adequate meal frequency was lower among children aged 6-23 months compared to those aged 24-59 months (AOR $=0.1,95 \% \mathrm{Cl}: 0.06,0.15)$ (Table 4). 
Table 4

Bivariate and multivariate analysis of factors associated with minimal meal frequency among children aged 6-59 months at Dabat HDSS site, northwest Ethiopia, 2016

\begin{tabular}{|c|c|c|c|c|}
\hline \multirow[t]{2}{*}{ Variables } & \multicolumn{2}{|c|}{ minimum meal frequency } & \multirow[t]{2}{*}{ Crude OR(95\% Cl) } & \multirow{2}{*}{$\begin{array}{l}\text { Adjusted OR } \\
(95 \% \mathrm{Cl})\end{array}$} \\
\hline & Adequate & Inadequate & & \\
\hline \multicolumn{5}{|l|}{ Child age } \\
\hline $6-23$ months & 352 & 165 & $0.1(0.05,0.13)$ & $0.1(0.06,0.15)^{\star \star}$ \\
\hline 24-59months & 633 & 24 & 1 & 1 \\
\hline \multicolumn{5}{|l|}{ Residence } \\
\hline Urban & 100 & 33 & $0.5(0.35,0.82)$ & $0.7(0.40,1.32)$ \\
\hline Rural & 885 & 156 & 1 & 1 \\
\hline \multicolumn{5}{|l|}{ ANC follow up } \\
\hline Yes & 642 & 135 & $0.7(0.53,1.05)$ & $1.1(0.76,1.66)$ \\
\hline No & 343 & 54 & 1 & 1 \\
\hline \multicolumn{5}{|l|}{ Time to initiation of CF } \\
\hline Timely initiation & 692 & 156 & $0.7(0.45,0.94)$ & $0.8(0.55,1.24)$ \\
\hline Not Timely initiation & 293 & 41 & 1 & 1 \\
\hline \multicolumn{5}{|l|}{ Preparation of CF } \\
\hline Prepared alone & 765 & 93 & $3.6(2.60,4.95)$ & $1.9(1.29,2.69)^{\star *}$ \\
\hline Prepared with other children & 220 & 96 & 1 & 1 \\
\hline \multicolumn{5}{|l|}{ Postnatal care services } \\
\hline Yes & 89 & 19 & $0.9(0.53,1.49)$ & $1.4(0.75,2.44)$ \\
\hline No & 896 & 170 & 1 & 1 \\
\hline
\end{tabular}

\section{Discussion}

The overall proportion of minimum dietary diversity among under five children that had at least one common childhood illness was $27 \%$ which was in lined with a finding in Eastern Ethiopia (25.2\%) [22] and Southeast Ethiopia (28.5\%) [23].The result was higher than national figure of dietary diversity (7\%) [14], Northwest Ethiopia (17\%) [24], Kenya (17.9\%) [25] and Southern Ethiopia (16\%)[26]. The discrepancy might be the national report included remote rural areas but in this study most of them (88.7\%) were from urban residence and have a chance to purchase food items and the report include children that had 
illness as well as healthy child but in this study all the study subjects have at least one common childhood illness during the study period and their mothers might have health information about increased food frequency and Varity during illness from health professionals. In addition, most of study subjects in northwest Ethiopia (87\%) were from rural residence and might not have access to buy Varity of food groups to improve dietary diversity [24].

The overall prevalence of minimum meal frequency was $83.9 \%$ which was higher than a study done in Southeast Ethiopia (68.4\%),[22], northwest Ethiopia (72.2\%),[23], Ghana (57.3\%)[24] Demographic and health survey report of Zimbabwe (59\%)[25], Malawi (53.5\%),[26] ,and Indonesia (58.2\%)[27]. The discrepancy might be due to a variation of dietary habit and culture. Furthermore $80 \%$ of study participants in southeast Ethiopia were healthier at time of data collection [22], whereas the current study participants have at least one common childhood illness during data collection period, thus the care givers might be encouraged for increasing meal frequency during illness and only $60 \%$ of the current study participants were breastfed child while $97 \%$ of study participant of northwest Ethiopia[23] and 91.6\% of study participants in southeast Ethiopia[22] were currently breastfed during the survey therefore the mothers might not increase meal frequency. The other difference might be most of the surveys in Asian countries used DHS data, which contain large populations with ethnic, cultural, and traditional variations on infant and young child feeding practices.

Mothers had ANC (Antenatal care) visit during pregnancy were more likely provided the recommended dietary diversity for their child compared with the counterpart This finding was supported by a study conducted in India [28]. This could be due to antenatal care service improves maternal counseling and community conversations program about the child feeding practices which enhance the understanding of mothers about how to prepare and feed their children with diversified foods. In addition, $31.8 \%$ of mothers who had ANC service provided the minimum recommended dietary diversity while only $17.9 \%$ of mothers who didn't had ANC service provided the minimum recommended dietary diversity thus ANC visits may become the key area to inform the care givers about the importance of providing diversified food groups to their children. Accordingly, intensified efforts are needed to improve the level of ANC service utilization for providing nutrition counseling to mothers on IYCF practices.

Mothers who purchased food source for households were more likely provide diversified food for their child compared with mothers obtained food source from home gardens. The finding was strengthened by a report, on which low income countries, like Ethiopia, the consumption of fruits and vegetables that are produced in home garden do not reach even one-third of the minimum recommended daily intake [29]. Furthermore most of the house hold produce monotonous food source that likely to be nutritionally inadequate in protein, fats and micronutrients to fill the recommended daily dietary intake [30].

The higher proportion of children who were delivered in the health institution were more likely provided diversified food than children were delivered at home. Similar findings have been reported by the previous studies done in east Africa region [31], southern Ethiopia [32] and northwest Ethiopia [33]. The possible explanation might mothers at post delivery period specifically the first 6 hours and 6 days might be 
counseled about the importance of timely initiation of complementary food and providing diversified food groups to their children [34]. Thus, intensified efforts are needed to improve the level of institutional delivery service utilization and provide nutrition counseling to mothers on IYCF practices.

The likelihood of feeding children with minimum dietary diversity was less among mothers who currently breast feed their children than the counterparts. Similar finding has been reported by a study conducted in Filipino [35]. The possible explanation might be, if the age of the child increases energy demand will be increased therefore, to achieve nutritional requirement, meal frequency will be increased. Furthermore most of the study subjects (88.7\%) were from rural resident and unable to read and write thus they might not considered that, if the child had breastfed, it is enough for his/her growth and development.

Children in the age group of 6-23 months were about less likely to receive the recommended meal frequency as compared to children in the age group of 24-59 months. The probably reason might be most of young children at this age have breastfed in order to attain nutritional requirements and prevent irreversible nutritional problem and mothers give special attention on breastfeeding rather than increasing frequency of complementary food. Furthermore, older children have an opportunity to eat food that may increase the meal frequency.

This study also indicated that; the likelihood of adequate meal frequency was higher among children whose mothers/care givers prepared food for the index child alone than mothers prepared food with other children. The explanation might be, if the number of children in the household increases, the food availability per head was frequently lower compared with households that have a single index child.

\section{Conclusion}

In this study, the proportion of children who received the minimum dietary diversity and meal frequency were low. ANC follow, household food source, breastfeeding and place of delivery were significantly associated with dietary diversity. Similarly, Age of children and ways of complementary food preparation had a significant association with meal frequency. Therefore, Improving the level of maternal and child health care utilization, providing nutrition counseling for mothers who use home gardening as a food sources and providing health and nutrition counseling on IYCF during maternal ANC service recommended for achieving the recommended dietary practices.

\section{Declarations}

Ethics approval and consent to participate

Ethical clearance was obtained from the Ethical Review Committee of the Institute of Public Health the University of Gondar (Ref: 2284/06/08). An official permission letter was obtained from the Dabat HDSS site, informed written consent was obtained from study participants in their local language after explaining the purpose of the study, potential risks and benefits of the study, and the right to withdraw from the study at any time. The participants were also assured that the data was confidential. 
Consent to publish

Not applicable

Availability of data and materials

Full data set and materials pertaining to this study can be obtained from corresponding author on reasonable request.

Competing interests

We the authors declare that there is no any competing interest.

Funding

No funding was obtained for this study.

\section{Authors' Contributions}

Authors' contributions: GA designed and supervised the study and ensured quality of the data and made a important contribution to the local implementation of the study. EA has cleaned and analyzed the data; as well as interpreted the results and drafted the manuscript. SS,KA,AK,MT,ZA,AT,ME,TA,AF,EG,YK, KA, AA and $\mathrm{KF}$ participated in analyzing and interpreting the data. GA, AT, MM, AF has critically reviewed the manuscript. All authors read and approved the final manuscript.

Authors' information

${ }^{1}$ Department of Human Nutrition, Institute of Public Health, University of Gondar, Gondar, Ethiopia

${ }^{2}$ Department of Health service, management and Health Economics, Institute of Public Health, University of Gondar, Gondar, Ethiopia

${ }^{3}$ Department of Epidemiology and biostatics, Institute of Public Health, University of Gondar, Gondar, Ethiopia

${ }^{4}$ Department of reproductive health, Institute of Public Health, University of Gondar, Gondar, Ethiopia.

Acknowledgements

We would like to thank university of Gondar for their financial and material support and mothers for their willingness to participate in the study.

\section{Abbreviations}


Antenatal Care

AOR

Adjusted Odd Ratio

COR

Crude Odd Ratio

EDHS

Ethiopian Demographic and Health Survey

HDSS

Dabat Health and Demographic surveillance System

IYCF

Infant and Young child feeding

SPSS

Statistical Package for Social Science

WHO

World Health Organization

\section{References}

1. Organization, W.H., Guiding principles for feeding non-breastfed children 6-24 months of age. 2005.

2. Organization, W.H., Indicators for assessing infant and young child feeding practices: part 1: definitions: conclusions of a consensus meeting held 6-8 November 2007 in Washington DC, USA. 2008.

3. Foote, J.A., et al., Dietary variety increases the probability of nutrient adequacy among adults. The Journal of nutrition, 2004. 134(7): p. 1779-1785.

4. Organization, W.H., Technical updates of the guidelines on the Integrated Management of Childhood IIIness (IMCI): evidence and recommendations for further adaptations. 2005.

5. Organization, W.H., Guiding principles for complementary feeding of the breastfed child. Geneva: WHO, 2003.

6. Stewart, C.P., et al., Contextualising complementary feeding in a broader framework for stunting prevention. Maternal \& child nutrition, 2013. 9: p. 27-45.

7. Jones, A.D., et al., W orld H ealth $O$ rganization infant and young child feeding indicators and their associations with child anthropometry: a synthesis of recent findings. Maternal \& child nutrition, 2014. 10(1): p. 1-17.

8. Brown, K.H., Diarrhea and malnutrition. The Journal of nutrition, 2003. 133(1): p. 328S-332S.

9. Neumann, C.G., M. Marquardt, and N.O. Bwibo, The impact of morbidity on food intake in rural Kenyan children. South African Journal of Clinical Nutrition, 2012. 25(3): p. 142-148.

10. Hug, L., D. Sharrow, and D. You, Levels \& trends in child mortality: report 2017. Estimates developed by the UN Inter-agency Group for Child Mortality Estimation. 2017. 
11. Rice, A.L., et al., Malnutrition as an underlying cause of childhood deaths associated with infectious diseases in developing countries. Bulletin of the World Health Organization, 2000. 78: p. 1207-1221.

12. UNION, A., The cost of hunger in Africa: social and economic impact of child undernutrition. 2012.

13. Haddad, L., et al., The Global Nutrition Report 2014: Actions and Accountability to Accelerate the World's Progress on Nutrition-. The Journal of nutrition, 2015. 145(4): p. 663-671.

14. Central Statistical Agency, Ethiopian Demographic and Health Survey report. 2016, CSA: Addis ababa,Ethiopia.

15. Brown, K., K. Dewey, and L. Allen, Complementary feeding of young children in developing countries: a review of current scientific knowledge. 1998.

16. Khanal, V., K. Sauer, and Y. Zhao, Determinants of complementary feeding practices among Nepalese children aged 6-23 months: findings from demographic and health survey 2011. BMC pediatrics, 2013. 13(1): p. 131.

17. Joshi, N., et al., Determinants of inappropriate complementary feeding practices in young children in Nepal: secondary data analysis of Demographic and Health Survey 2006. Maternal \& child nutrition, 2012. 8: p. 45-59.

18. The Sustainable Development Goals (SDGs), 2015-2030. 2015, United Nation.

19. Federal Ministry of Health Family Health Department Ethiopia, NATIONAL STRATEGY FOR INFANT AND YOUNG CHILD FEEDING. 2004: Addis ababa.

20. Federal Ministry of Health, National Nutrition Program. 2008: Addis ababa,Ethiopia.

21. Ethiopian Health and Nutrition Research Institute, NUTRITION BASELINE SURVEY REPORT FOR THE NATIONAL NUTRITION PROGRAM OF ETHIOPIA. 2010: Addis Ababa.

22. Tegegne, M., et al., Factors associated with minimal meal frequency and dietary diversity practices among infants and young children in the predominantly agrarian society of Bale zone, Southeast Ethiopia: a community based cross sectional study. Archives of Public Health, 2017. 75(1): p. 53.

23. Belew, A.K., et al., Dietary diversity and meal frequency among infant and young children: a community based study. Italian journal of pediatrics, 2017. 43(1): p. 73.

24. Saaka, M., et al., Magnitude and factors associated with appropriate complementary feeding among children 6-23 months in northern Ghana. BMC Nutrition, 2016. 2(1): p. 2.

25. Zimbabwe National Statistics Agency, Mulitiple indicator cluster survey. 2014: Harare, Zimbabwe.

26. National Statistical Office Zomba, M., Malawi Demographic and Health Survey 2010.

27. Sciences, I.I.I.f.L., Food and dietary diversity in the context of food and nutrition security in Indonesia. 2014.

28. Malhotra, N., Inadequate feeding of infant and young children in India: lack of nutritional information or food affordability? Public health nutrition, 2013. 16(10): p. 1723-1731.

29. Darnton-Hill, I. and B. Cogill, Maternal and young child nutrition adversely affected by external shocks such as increasing global food prices. The Journal of nutrition, 2009. 140(1): p. 162S-169S. 
30. Cabalda, A.B., et al., Home gardening is associated with Filipino preschool children's dietary diversity. Journal of the American Dietetic Association, 2011. 111(5): p. 711-715.

31. Gewa, C. and T. Leslie, Young child complementary food diversity in the East African region: crosscountry differences, geographic distribution and relationship with health-care utilization. 2013, Federation of American Societies for Experimental Biology.

32. Gatahun, A. and M. Abyu, Dietary diversity feeding practice and determinants among children aged 623 months in Kemba Woreda, southern Ethiopia implication for public health intervention. Nutrition \& Food Sciences, 2015.

33. Temesgen, H., T. Yeneabat, and M. Teshome, Dietary diversity and associated factors among children aged 6-23 months in Sinan Woreda, Northwest Ethiopia: a cross-sectional study. BMC Nutrition, 2018. 4(1): p. 5.

34. Kennedy, E., et al., Multisector nutrition program governance and implementation in Ethiopia: opportunities and challenges. Food and nutrition bulletin, 2015. 36(4): p. 534-548.

35. Wright, M.J., et al., The interactive association of dietary diversity scores and breast-feeding status with weight and length in Filipino infants aged 6-24 months. Public health nutrition, 2015. 18(10): p. 1762-1773.

\section{Figures}




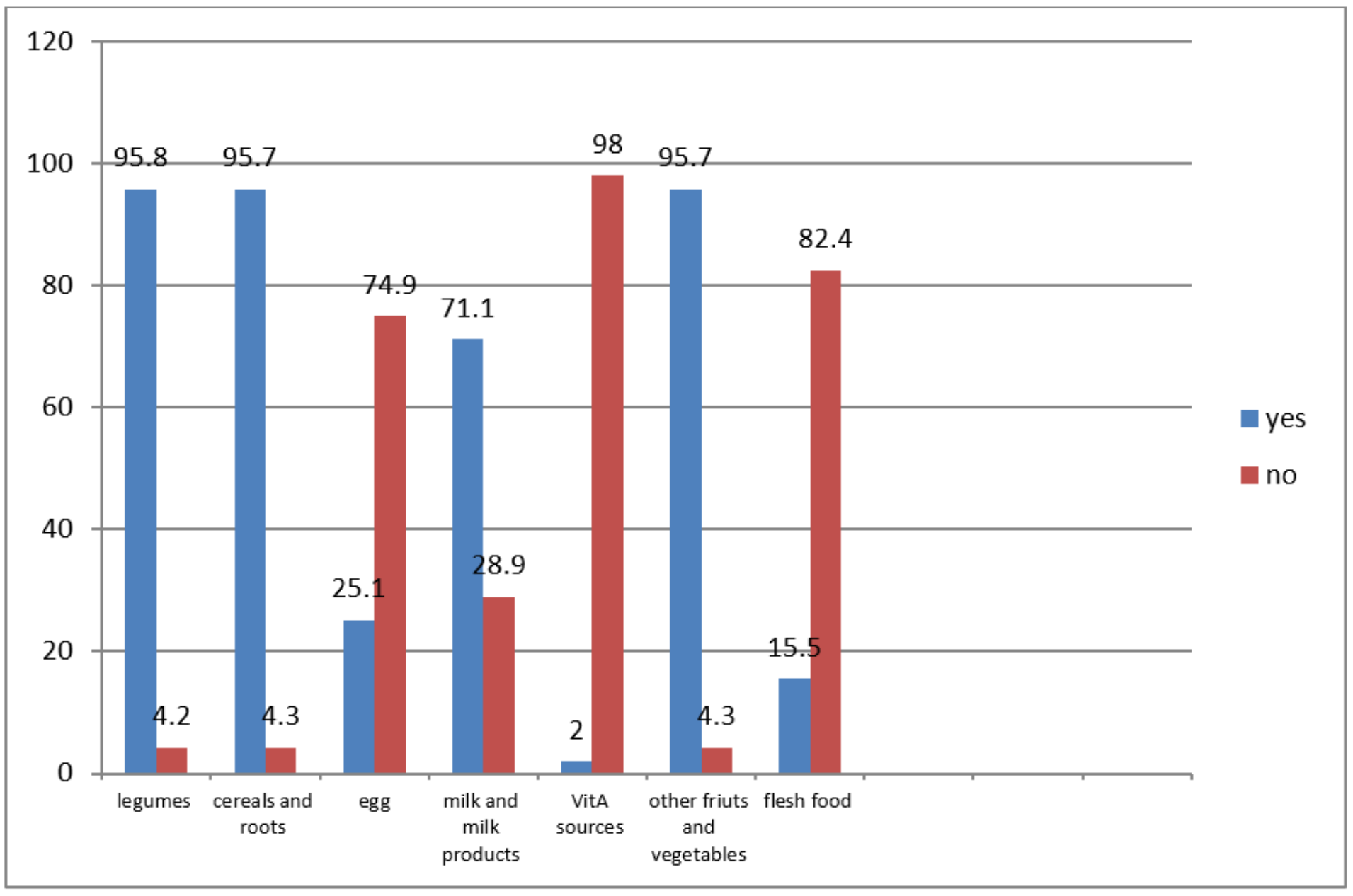

Figure 1

Types of food groups given to children aged 6-59 months old, Dabat HDSS site, northwest Ethiopia. 